\title{
Wind Power with Energy Storage Arbitrage in Day-ahead Market by a Stochastic MILP Approach
}

\author{
I.L.R. GOMES, Departamento de Física, Escola de Ciências e Tecnologia, \\ Universidade de Évora, 7000-645 Évora, Portugal, ICT, Instituto de Ciências da \\ Terra, Universidade de Évora, 7000-645 Évora, Portugal, IDMEC, Instituto \\ Superior Técnico, Universidade de Lisboa, 1049-001 Lisbon, Portugal.
}

R. MELICIO*, Departamento de Física, Escola de Ciências e Tecnologia, Universidade de Évora, 7000-645 Évora, Portugal, ICT, Instituto de Ciências da Terra, Universidade de Évora, 7000-645 Évora, Portugal, IDMEC, Instituto Superior Técnico, Universidade de Lisboa, 1049-001 Lisbon, Portugal.

V.M.F. MENDES, Departamento de Física, Escola de Ciências e Tecnologia, Universidade de Évora, 7000-645 Évora, Portugal, Department of Electrical Engineering and Automation, Instituto Superior de Engenharia de Lisboa, 1959-007 Lisbon, Portugal, CISE, Electromechatronic Systems Research Centre, Universidade da Beira Interior, 6201-001 Covilhã Portugal.

H.M.I. POUSINHO, Departamento de Física, Escola de Ciências e Tecnologia, Universidade de Évora, 7000-645 Évora, Portugal.

\begin{abstract}
This paper is about a support information management system for a wind power (WP) producer having an energy storage system (ESS) and participating in a day-ahead electricity market. Energy storage can play not only a leading role in mitigation of the effect of uncertainty faced by a WP producer, but also allow for conversion of wind energy into electric energy to be stored and then released at favourable hours. This storage provides capability for arbitrage, allowing an increase on profit of a WP producer, but must be supported by a convenient problem formulation. The formulation proposed for the support information management system is based on an approach of stochasticity written as a mixed integer linear programming problem. WP and market prices are considered as stochastic processes represented by a set of scenarios. The charging/discharging of the ESS are considered dependent on scenarios of market prices and on scenarios of WP. The effectiveness of the proposed formulation is tested by comparison of case studies using data from the Iberian Electricity Market. The comparison is in favour of the proposed consideration of stochasticity.
\end{abstract}

Keywords: Electricity markets, energy storage, mixed integer linear programming, stochastic optimization, wind power

*E-mail: ruimelicio@gmail.com

Vol. 00, No. 0, (C) The Author(s) 2019. Published by Oxford University Press. All rights reserved. For permissions, please e-mail: journals.permission@oup.com. 


\section{Introduction}

The conference on climate change held in Paris in December 2015 adopted a new treaty signed by 195 nations aiming at limiting climate change to below $2{ }^{\circ} \mathrm{C}$, implying the reduction in the usage of the conventional technology for energy conversion based in fossil fuel [17]. Conversion technology based in renewable energy sources (RES) are alternatives and the alternatives favoured are wind power (WP) and photovoltaic (PV) power due to an impressive reduction in costs, achieving parity with conventional technology. For instance, PV prices dropped more than $60 \%$ between 2009 and 2016 [32]. Exploitation of RES matches a supply of about $23 \%$ of total electricity generation in 2015 [29]. Progress in WP and PV power pushed up the growth of the participation of renewable energy in the mix of energy conversion to a record of exceeding $150 \mathrm{GW}$ in 2015, according to the International Energy Agency [29]. But large-scale integration either WP or PV sources per se are a menace due to the fact of not being capable of ensuring the desired continuing on power conversion to satisfy the demand. The intermittence and variability on the availability of the respective energy sources is a challenge to be faced for the satisfaction of the demand while providing reliable services.

Large-scale integration of WP and PV power increases the need for flexibility options [10]. The challenge associated with the meeting of the satisfaction of demand while providing reliable services has renewed the interest in research and development of energy storage systems (ESS). Particularly, RES integration without energy storage is viewed as tough integration for future power systems [9]. Hence, energy storage can play a leading role in the actual and future deregulated markets, providing capability for arbitrage, contributing to a better management by increasing the profit of a producer exploiting RES having intermittences [11].

\section{Relationship to Energy 4.0}

A new term regarding the sector of energy of the future is emerging-Energy 4.0 [23]. Energy 4.0 branches from the all-embracing term industry 4.0 [8]. The availability of the data to aid in decision for the energy sector is ongoing and will play an important role in the efficiency, reliability, security and cyber security of smart grids [5-7] in the context of Energy 4.0. For example, the efficiency, reliability and security of a WP system having an ESS as a way to harness RES is improved with the availability of data from weather conditions allowing more convenient management of charge and discharge of energy. The change of pattern from the traditional electric grid to a smart electric grid [4] paved the way for the future in the way of power systems enhancement. Intelligence in the form of new computing models, algorithms and advanced analytics is prone to enable enhancement in decision making [13]. A smart system can be stated as an embedded system that incorporates advanced systems and provides sophisticated monitoring and control over how something happens in the system [12]. Power systems are extensively handlers of intelligence in the scope of Energy 4.0. But, menaces and challenges to the former energy conversion into electric energy are appearing at an unprecedented speed due to the increasing of exploitation of intermittent RES, the need for augmenting the capability of bidirectional flow of energy in the lines of electric grids and the need to accommodate significant capacity of energy storage in sustainable and economic ways. Also, the business regarding the sector of electric energy has changed at unprecedented ways: new market players; new products; new tools to process the huge collection of data to support decisions in due time, for instance, a survey on data mining techniques applied to energy time series forecasting is presented in [27]; and new methodology in energy trading for better decision-making. Therefore, monitoring and high-quality real time data is needed for a better exploitation of WP. 


\section{State of the Art}

Although WP can be forecasted using time series [19, 26], artificial intelligence [1, 24, 25] and ensemble models [31], the uncertainty on WP has an influence that cannot be discarded in the bidding for participation in the day-ahead market (DAM). Hence, the uncertainty on WP must be properly accommodated and the accommodation can be made by considering the WP availability as a stochastic process or by having enough energy available to cover the uncertainty in due time.

A WP producer is subjected to the uncertainty on availability of WP, so one expects that the bid accepted at the clearing of DAM is not necessarily in accordance with the production to be delivered in due time in the next day. This non-accordance is called imbalance. So, the decision-making for bidding of a WP producer due to uncertainty needs a support management system to overcome as much as possible the losses of profit due to energy imbalance incurred by the power producer at the time of delivering, i.e., the operation time. Research efforts made for aiding the participation of WP producers in electricity markets can be classified in developments embracing three main lines. The first line explores the WP producer participation in DAM by the use of stochastic optimization, for instance, with the goal of minimizing the expected costs of imbalances in the operation time [28]. This first line involves the consideration on a single period of the optimization problem several possible scenarios of WP production and imbalance penalty costs. The second line explores the WP producer ability to convert the electrical energy in other types of energy that can be stored and released at favourable hours to cover the uncertainty in due time $[2,16,30]$. This line also must deal with the uncertainty in an economic point of view. The third line explores the use of financial options as a tool for a WP producer to hedge against WP uncertainty, covering imbalances in the operation time in an economic point of view [18]. This paper embraces the first line of research, a maximization of the expected profit by a stochastic mixed integer linear programming (MILP) problem. MILP has been an approach with success for aiding in the trading of the energy in electricity markets $[20,21]$ and has powerful commercial solvers available. A model of an ESS based in a vanadium redox flow battery (VRFB) [3] is integrated in the problem formulation to provide for arbitrage. The VRFB is one of the most promising technology to mitigate the consequences of intermittence of the RES. In addition, the paper presents a formulation for charge or discharge of the ESS according to the scenarios for the DAM prices and for the WP. The problem formulation proposed is addressed for a context of DAM inspired by the rules of the Iberian Electricity Market (MIBEL). MIBEL is a pool-based market where all market players must submit the bids for a whole day at 10 a.m. of the previous day. The accepted bids programme defines the unit commitment for the 24 hours of the next day. The marginal price and the volumes of energy are established for the whole market by a matching between the offers of purchasing and of selling. However, the market players in MIBEL can present new bids during the day of energy delivery till at least 3 hours before the operation time to the market called intraday market; this intraday market deserves an appropriated approach. So, the intraday market is not in the scope of this paper.

\section{DAM and Balancing Market}

The uncertainty on availability of WP subjects the producer to be in a situation of eventual real time non-compliance with the bid accepted at the clearing of DAM, i.e., the availability of the energy source to deliver the production in due time in the operation time of the next day is not enough or exceeds the bid accepted. The system operator uses a procedure of economic penalty for convenient addressing the keeping of the balance between demand of energy and generation to cope with the DAM imbalance. 
The system operator at the balancing market of MIBEL subjects the producer to a price for the positive energy imbalance and another price for negative energy imbalance [14, 15, 22]. In what follows, the energy imbalance incurred by a power producer owning a power plant $k$ in period $t$ is stated as follows:

$$
d_{t}^{k}=P_{t s}^{k}-P_{t}^{k}
$$

In (1), $P_{t s}^{k}$ is the physical delivering of energy in period $t$ and $P_{t}^{k}$ is the energy submitted bid in the DAM. A positive or negative energy imbalance of a physical energy delivering in the operation time is associated with a positive or negative imbalance price $\lambda_{t}^{+}$and $\lambda_{t}^{-}$, respectively. If the power producer has an excess of energy, the remaining energy can be sold in the balancing market at price $\lambda_{t}^{+}$. If the power producer has a deficit of energy, the energy in deficit should be purchased in the balancing market $\lambda_{t}^{-}$. The imbalance prices are usually defined by means of price ratios as follows:

$$
\begin{aligned}
& p r_{t}^{+}=\frac{\lambda_{t}^{+}}{\lambda_{t}}, \quad p r_{t}^{+} \leq 1 \\
& p r_{t}^{-}=\frac{\lambda_{t}^{-}}{\lambda_{t}}, \quad p r_{t}^{-} \geq 1 .
\end{aligned}
$$

In (2) and (3), $\lambda_{t}$ is the DAM price and $p r_{t}^{+}$and $p r_{t}^{-}$are respectively the positive and negative imbalance price ratios. Consequently, a producer $k$ has a profit at period $t$ given as follows:

$$
P R_{t}^{k}=\lambda_{t} P_{t}^{k}+\left(\lambda_{t} p r_{t}^{+} d_{t}^{k+}-\lambda_{t} p r_{t}^{-} d_{t}^{k-}\right)
$$

In (4), the first term represents the profit associated with the DAM price $\lambda_{t}$ from the accepted production $P_{t}^{k}$. The term in brackets is associated with the income due to the imbalances derived from the procedure of economic penalty. The positive and negative deviations are respectively given by $d_{t}^{k+}$ and $d_{t}^{k-}$ and are subjected to the constraint of at least one of the deviations is always null at any period $t$. However, this procedure of economic penalty is in accordance with the MIBEL; the procedure of economic penalty can easily be customized to other rules for DAMs.

\section{Problem Formulation}

The objective of the optimization problem is maximization of an objective function given by the expected profit. So, to achieve this objective, a convenient consideration of the economic impact of imbalance must be integrated in the problem formulation. This convenient consideration must anticipate the economic consequence of uncertainty on market prices and on availability of WP in the operation time to be converted into electric energy. If there is a mismatch between the energy bid accepted at the close of the DAM and the actual production, then eventual losses on profit can occur.

The proposed problem formulation is a sort of a two-stage stochastic optimization problem where the hourly bids and imbalances are first- and second-stage variables, respectively. Two-stage stochastic programming is characterized by considering decisions made in two stages: first-stage, having decisions made before the realization of the random variables; and second-stage, having decisions made after knowing the realization of random variables and depending on the decisions made in first-stage. Generally, the two-stage stochastic MILP formulated in terms of a continuous random vector which accounts for all the uncertain parameters of the problem is numerically intractable. But, to cope with intractability, the original random vector can be approximate by one with a finite number of realizations or scenarios, the scenario tree. The uncertainty on WP 
availability and on market prices are modelled by a convenient selection of scenarios from historical data giving the set $S$ said to be the set of appropriate scenarios for the next day. Together with a scenario $s$ is associated a probability $\pi_{s}$ of occurrence, where $\pi_{s}=1 / S$. The indexing to the power plant $k$ for purpose of simplicity is not explicitly presented in what follows.

\subsection{Coordinated bidding with stochastic management of ESS}

The stochastic MILP formulation is given as follows:

$$
\max \text { PROFIT }=\sum_{s=1}^{S} \sum_{t=1}^{T} \pi_{s}\left(\lambda_{t s} P_{t}+\lambda_{t s} p r_{t s}^{+} d_{t s}^{+}-\lambda_{t s} p r_{t s}^{-} d_{t s}^{-}\right) .
$$

General constraints:

a) Energy offer constraint

$$
0 \leq P_{t} \leq P^{W \max }+P_{t}^{D i E S S \max } .
$$

b) Output power of WP and ESS

$$
P_{t s}=P_{t s}^{W}-P_{t s}^{C h E S S}+P_{t s}^{D i E S S} .
$$

c) Imbalance constraints

$$
\begin{gathered}
d_{t s}=P_{t s}-P_{t}, \forall t, \forall s \\
d_{t s}=d_{t s}^{+}-d_{t s}^{-}, \forall t, \forall s \\
0 \leq d_{t s}^{+} \leq P_{t s} u_{t s}, \forall t, \forall s \\
0 \leq d_{t s}^{-} \leq\left(P^{W \max }+P^{D i E S S \max }\right)\left(1-u_{t s}\right), \forall t, \forall s .
\end{gathered}
$$

In (5), if the imbalance is negative, the term $\lambda_{t s} p r_{t s}^{+} d_{t s}^{k+}$ is null and the term $\lambda_{t s} p r_{t s}^{-} d_{t s}^{k-}$ is subtracted; if the imbalance is positive, the term $\lambda_{t s} p r_{t s}^{-} d_{t s}^{k-}$ is null and the term $\lambda_{t s} p r_{t s}^{+} d_{t s}^{k+}$ is added. In (6), the limit on the bid is set to be the rated power of the wind system plus the maximum discharge power of ESS. In (8-11), the imbalance is decomposed into the difference between the $d_{t s}^{k+}$ and the $d_{t s}^{k-}$ imbalances.

Constraints of ESS:

a) Energy storage equation

$$
E_{t s}^{E S S}=E_{t-1, s}^{E S S}+\eta^{C h E S S} P_{t s}^{C h E S S}-\frac{1}{\eta^{\text {DiESS }}} P_{t s}^{D i E S S} .
$$

b) Energy storage limits

$$
0 \leq E_{t s}^{E S S} \leq E^{E S S \max }
$$

c) Storage power limits

$$
\begin{aligned}
& 0 \leq P_{t s}^{C h E S S} \leq P_{t s}^{C h E S S \max } k_{t s} \\
& 0 \leq P_{t s}^{D i E S S} \leq P_{t s}^{D i E S S \max }\left(1-k_{t s}\right) .
\end{aligned}
$$


In (12), the balance of energy is imposed between sequential time periods on the ESS: $E_{t s}^{E S S}$ and $E_{t-1, S}^{E S S}$ are at scenario $s$ the energy stored at period $t$ and period $t-1$, respectively; $\eta^{C h E S S}$ and $\eta^{D i E S S}$ are the efficiency of charging and discharging, respectively; and $P_{t s}^{C h E S S}$ and $P_{t s}^{D i E S S}$ are the power of charge and discharge at period $t$ and at scenario $s$, respectively. The balance of energy in the ESS in (12) assumes a null depth of discharge, normally assumed when modelling the type of energy storage associated with a VRFB. In (13), the limit on the energy storage is set to be the rated power of ESS. In (14) and (15), the charging and discharging constraints on ESS are imposed: if the ESS is charging, the binary variable $k_{t s}$ is set to 1 and (14) defines a box constraint for the charging power, while discharging is set to a null value due to (15) and if the ESS is discharging, (15) defines a box constraint for the discharging power, while charging is set to a null value due to (14).

The problem formulation above is the stochastic management proposed for the bidding coordinated with the ESS to support the decision of the producer in the DAM. This formulation can be easily customized for the case of uncoordinated bidding or the case of coordinated bidding with deterministic management of ESS. These customizations allow a comparison with the proposed stochastic management and are as follows:

\subsection{Uncoordinated bidding}

Uncoordinated bidding is performed by setting all the parameters and variables related to the ESS to zero in the formulation (5-15), for instance, $P_{t s}^{C h E S S}$ and $P_{t s}^{D i E S S}$ to be zero for all $t$ and $s$.

\subsection{Coordinated bidding with deterministic management of ESS}

Coordinated bidding with deterministic management of ESS is performed by replacing all the variables related to the ESS by the respective variables without the consideration of stochasticity in the formulation (5-15), for instance, $P_{t s}^{C h E S S}$ and $P_{t s}^{D i E S S}$ are replaced by $P_{t}^{C h E S S}$ and $P_{t}^{D i E S S}$.

The optimization of WP producer participation in a DAM having an ESS is in accordance with the diagram of flow shown in Figure 1.

In Figure 1, the upper blocks are for scenario generation procedures obtained via historical data of WP and historical data of MIBEL taken as input data in the scope of this paper. Then, technical data blocks access the wind and ESS specification to deliver the information into the block implementing the two-stage stochastic problem formulation by the MILP approach solved by GAMS/CPLEX.

\section{Case Studies}

\subsection{Input data and case studies}

The data of the DAM of MIBEL are used for an exploitation of WP in Portugal with a wind farm having a rated power of $100 \mathrm{MW}$ and an ESS rated power of $30 \mathrm{MW}$. The bidding is on an hourly basis. The scenarios for the hourly DAM prices and for the positive imbalance from data 10 days of June 2015 are respectively shown in Figure 2.

The scenarios for negative imbalance prices and for the hourly WP scenarios for the respectively 10 scenarios are respectively shown in Figure 3.

In Figures 2 and 3, the historical data shown for the hourly DAM prices and the hourly imbalance price scenarios are reported in [33]. Each set of DAM prices of imbalance prices or of WP has 10 scenarios that are usually considered equiprobable ones in each set. But a different probability for scenarios can be considered. The case studies are organized as shown in Table 1. 


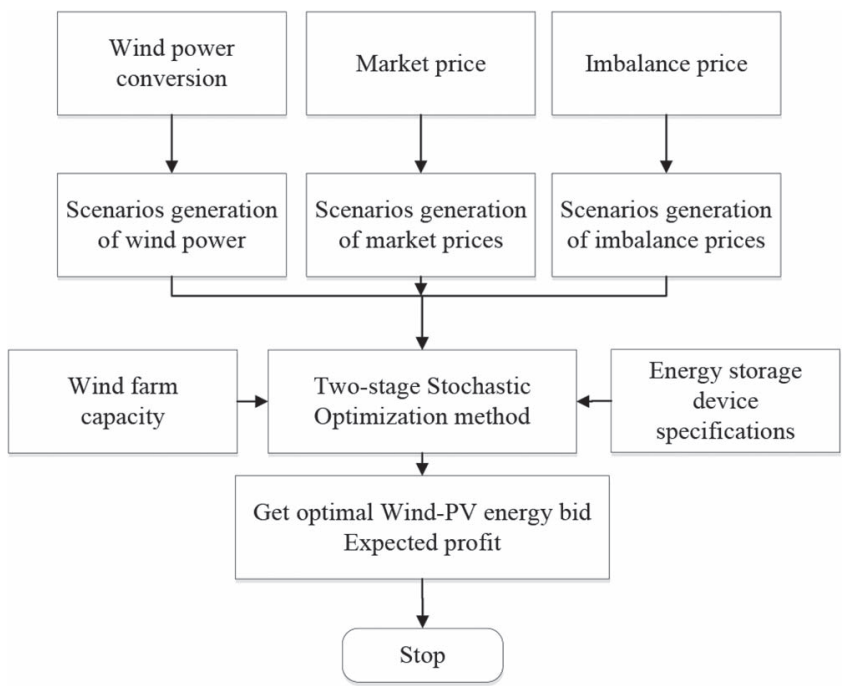

FIGURE 1 Bidding strategy for WP producer having ESS.
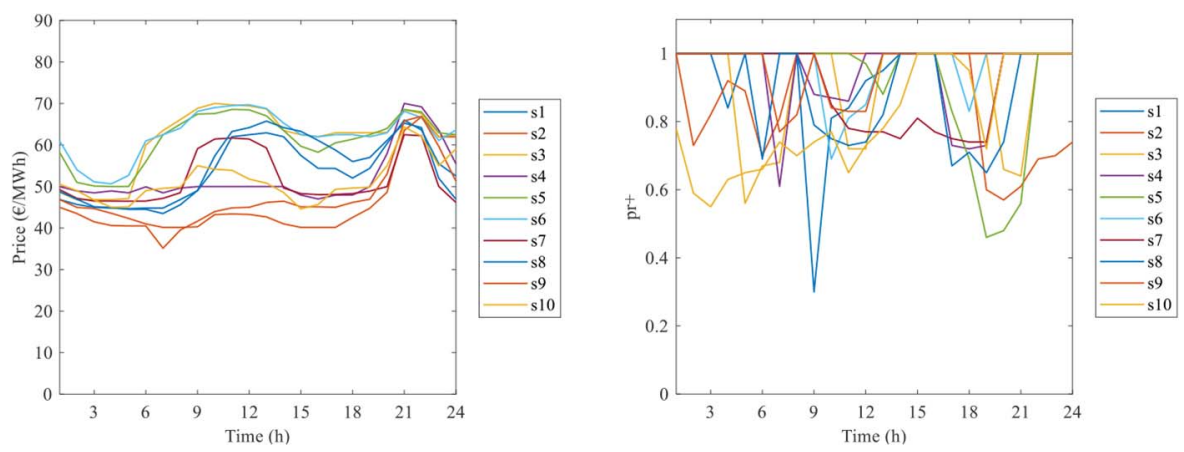

FIGURE 2 Left: hourly DAM prices; rigth: positive imbalance prices.
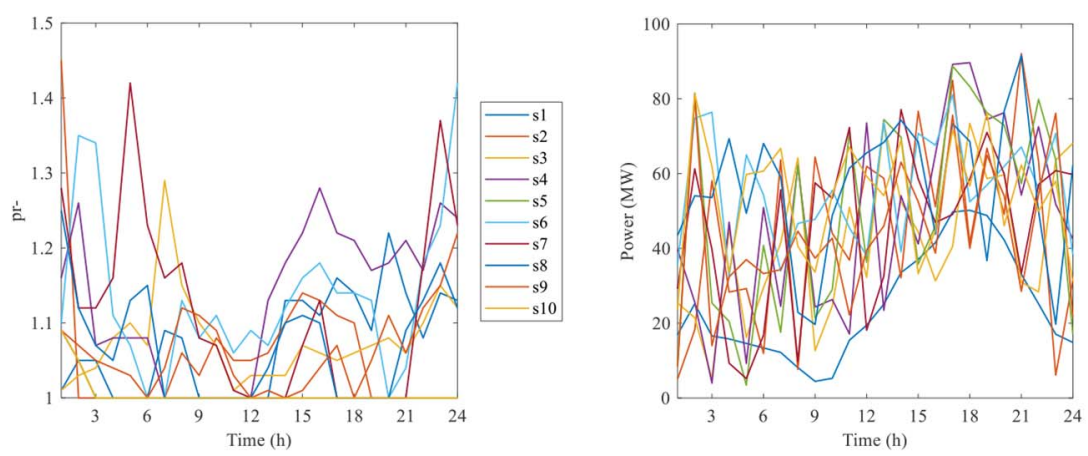

FIGURE 3 Left: negative imbalance price; rigth: WP scenarios. 
8 Wind Power with Energy Storage Arbitrage in Day-ahead Market by a Stochastic MILP Approach

TABLE 1 Case studies description

\begin{tabular}{ll}
\hline Case & Description \\
\hline Case \#1 & Uncoordinated bidding \\
Case \#2 & Coordinated bidding with deterministic management of ESS \\
Case \#3 & Coordinated bidding with stochastic management of ESS \\
\hline
\end{tabular}

TABLE 2 Case studies characteristics

\begin{tabular}{llll}
\hline$\#$ & Case \#1 & Case \#2 & Case \#3 \\
\hline CPU solution time (s) & 4 & 6 & 7 \\
Number of equations & 24,745 & 24,793 & 38,401 \\
Continuous variables & 24,769 & 24,841 & 38,665 \\
Integer variables & 240 & 264 & 2,640 \\
\hline
\end{tabular}

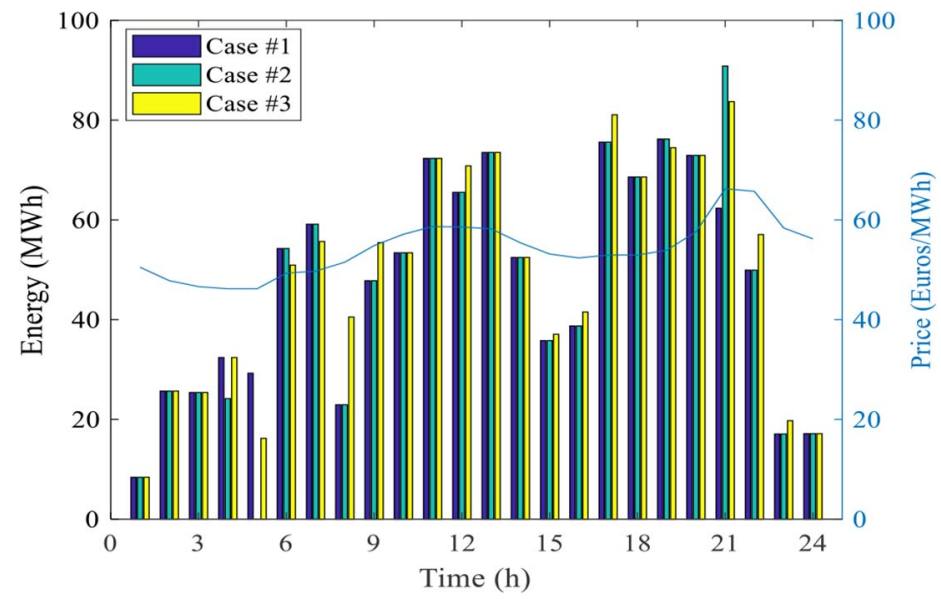

FIGURE 4. Energy traded and average hourly DAM price.

\subsection{Simulation results}

The proposed formulations have been implemented in GAMS, using the commercial solver CPLEX 10.0 to solve the MILP approach in accordance with the above description for Cases \#1-3. The numerical simulations are performed on a 1.6-GHz-based processor with 4 GB of RAM. The characteristics concerning the CPU time, number of equations, continuous variables and integer variables of each case study are shown in Table 2.

In Table 2, it is shown that the CPU solutions time allows for various simulations that can be useful for testing different sets of scenarios, i.e., less than $10 \mathrm{~s}$ of CPU solution time is most appropriate for the practical application of this support information management system. The bidding and the average hourly DAM price for Cases \#1-3 are shown in Figure 4.

In Figure 4, it is shown in accordance with the three case studies that the energy traded as expected is influenced by the DAM prices. Cases \#2 and 3 show a tendency for higher levels in hours of likely high market prices, for instance, between 21 and 23 hours. A comparison of Case \#3 with the other 


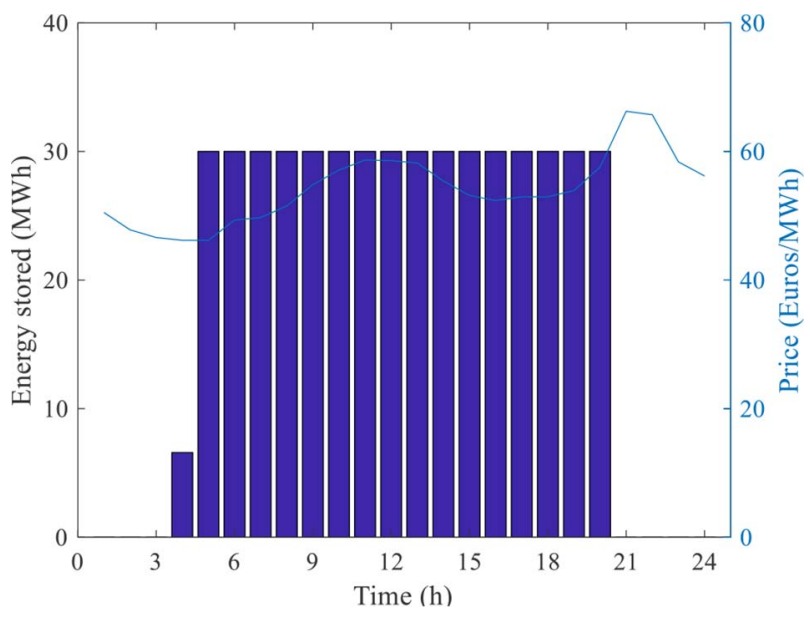

FIGURE 5. Case \#2: energy stored.

cases shows a tendency for an increase in the level of energy bidding at hours not necessary of likely high DAM prices and shows a trade of energy increase in more than $4.9 \%$ in comparison with Case \#2. The level of energy in storage in Case \#2 and the average hourly DAM price are shown in Figure 5.

In Figure 5, it is shown that although there is a $24 \%$ loss of energy of a round trip energy in the ESS, i.e., of a cycle of charge and discharge, the ESS is called for achievement of a more favourable bidding. The ESS charges in Hours 4 and 5, as expected, due to the likely low DAM prices and favourable positive imbalance prices. Also, as expected, higher charge is made at the lowest likely price at Hour 5. This ESS management favours a convenient accommodation of energy, storing and discharge at Hour 21, i.e., at a more profitable hour. The Case \#2 profit is about $59.7 \mathrm{k} €$. But, the consideration of uncertainty in the energy balance equation of the ESS achieves a better profit of about $59.9 \mathrm{k} €$. Case \#3 for the values scenarios at Hours 4 and 5 has the charged energy in function of the DAM prices and the WP as shown in Figure 6.

Case \#3 for the values scenarios at Hours 21 and 22 has the discharged energy in function of the DAM prices and the WP as shown in Figure 7.

In Figure 6, it is shown that at Hour 4, for the Scenarios 5-10 of WP and for all scenarios of DAM price at these hours, the charging is disadvantageous except at the combination WP Scenario 8/Price Scenario 6 where the ESS is fully charged. Contrary to Hour 4, at Hour 5 for the Scenarios 6-10 of WP and for all scenarios of hourly DAM price, the energy charging is advantageous except at the combination WP Scenario 8/DAM Price Scenario 6 and at the combinations WP Scenario 7/all hourly DAM price scenarios where the ESS is never charged. Figure 7 shows that at Hour 21, for the Scenarios 4 and 5 of WP and for all scenarios of hourly DAM price, the energy discharged is advantageous. Contrary to Hour 21, at Hour 22 for the Scenarios 4-7 of WP and for all scenarios of hourly DAM price, the energy discharged is disadvantageous. Figure 6 and Figure 7 are in favour of the conclusion that the discarding of the uncertainty drives managements for the ESS which although optimum for the realization of a particular scenario are not necessarily in compliance with what can happen during operation time. Case \#3 gives the decision maker a better support to decide when and the level of energy to charge or discharge in accordance with the uncertainty on DAM price scenarios and on the WP scenarios. The expected profit for each case study is shown in Table 3. 
10 Wind Power with Energy Storage Arbitrage in Day-ahead Market by a Stochastic MILP Approach
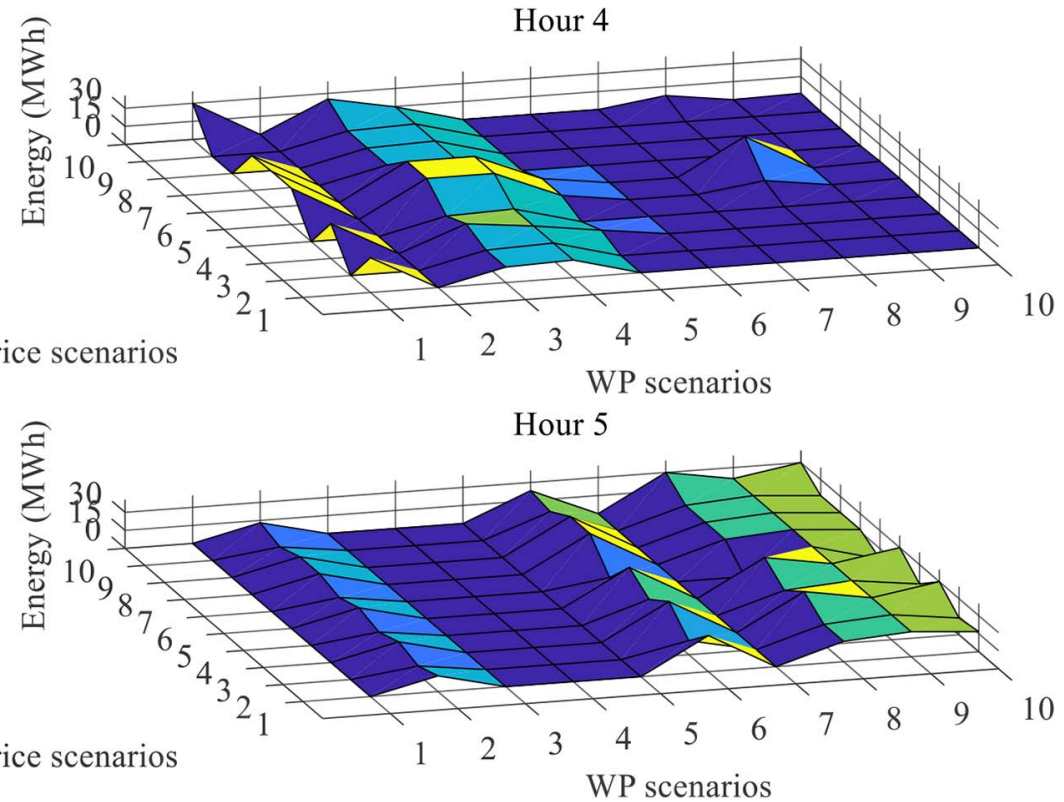

FIGURE 6. Case \#3: energy charged as function of WP scenarios and hourly DAM price scenarios.
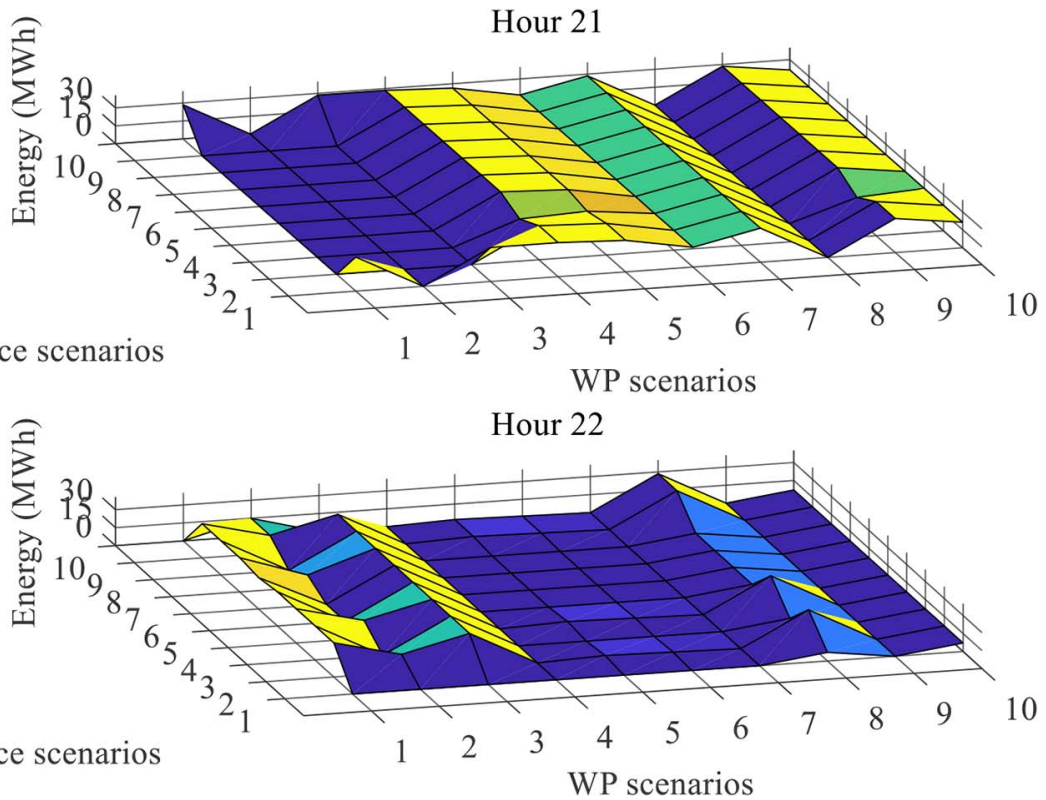

FIGURE 7. Case \#3: energy discharged as function of WP scenarios and hourly DAM price scenarios. 
TABLE 3 Expected profit for each case study

\begin{tabular}{ll}
\hline Case & Expected profit $(\mathrm{k} €)$ \\
\hline Case \#1 & 59.590 \\
Case \#2 & 59.746 \\
Case \#3 & 59.882 \\
\hline
\end{tabular}

In Table 3, it is shown that Case \#2 allows an increment in the expected profit in comparison with Case \#1 and Case \#3 even allows a higher increment in the expected profit. Therefore, to this set of case studies is expected in general that the consideration of scenarios is a better management than the deterministic one to hedge against uncertainty. The capability given by support information management system in aiding in the decision of charge or discharge of the ESS according to the scenarios of prices and WP is shown to be a valuable economic asset.

\section{Conclusion}

Stochastic optimization is suitable for problems needing to consider uncertainty in the input data, as the instance of problem concerned with the context of bidding in DAM for WP producers. Market energy prices and imbalance prices can be favourable, modelled by a set of scenarios driven from historical data. In this context, a problem formulation for a support information management system considering stochasticity is proposed to aid a WP producer with an ESS participating with bids in a DAM. ESS can provide the capability of arbitrage, but this arbitrage must be in compliance with the uncertainty on prices and on WP to be a valuable economic asset. The formulation in accordance with this compliance considers the dependence of the equation of balance of ESS with the scenarios of DAM prices and WP. Hence, the decision to charge or discharge the ESS takes into consideration the influence of both scenarios of DAM price and WP. The formulation is conveyed to be treated by a MILP approach allowing the use of integer variables, for instance, needed for modelling the ESS in what regards charging/discharging of energy. MILP is further favourable for practical applications due to the excellent commercial solvers available. A comparison of case studies using data for WP in Portugal with DAM prices from the MIBEL is addressed for uncoordinated and coordinated bids with deterministic or stochastic management of the ESS. This comparison allows to conclude that a better management for exploitation of WP with arbitrage is achieved with the stochastic management of the ESS.

\section{Acknowledgments}

We thank the Bolsas Camões, I.P. and Millennium BCP Foundation for the financial support. This work is funded by European Union through the European Regional Development Fund, included in the COMPETE 2020 (Operational Program Competitiveness and Internationalization) through the ICT project (UID/GEO/04683/2019) with the reference POCI010145FEDER007690; Portuguese Foundation for Science and Technology (FCT) under Project UID/EEA/04131/2019; and FCT, through IDMEC, under LAETA, Project UID/EMS/50022/2019. 
12 Wind Power with Energy Storage Arbitrage in Day-ahead Market by a Stochastic MILP Approach

\section{References}

[1] N. Amjady, F. Keynia and H. Zareipour. Wind power prediction by a new forecast engine composed of modified hybrid neural network and enhanced particle swarm optimization. IEEE Transaction on Sustainable Energy, 2, 265-276, 2011.

[2] J. L. Angarita, J. Usaola and J. Martínez-Crespo. Combined hydro-wind generation bids in a pool-based electricity market. Electric Power Systems Research, 79, 1038-1046, 2009.

[3] B. N. Arribas, R. Melício, J. G. Teixeira and V. M. F. Mendes. Vanadium redox flow battery storage system linked to the electric grid. Renewable Energy and Power Quality Journal, 1(14), 1025-1030. 2016.

[4] T. Arsan. Smart systems: from design to implementation of embedded smart systems. In IEEE International Symposium on High Capacity Optical Networks and Enabling Technologies, Nicosia, Cyprus, pp. 59-64. 2016.

[5] N. C. Batista, R. Melicio, J. C. O. Matias and J. P. S. Catalão. ZigBee standard in the creation of wireless networks for advanced metering infrastructures. In IEEE 16th Mediterranean Electrotechnical Conference, Medina Yasmine Hammamet, Tunisia, pp. 220-223. 2012.

[6] N. C. Batista, R. Melicio, J. C. O. Matias and J. P. S. Catalão. Photovoltaic and wind energy systems monitoring and building/home energy management using ZigBee devices within a smart grid. Energy, 49, 306-315, 2013.

[7] N. C. Batista, R. Melício and V. M. F. Mendes. Layered smart grid architecture approach and field tests by ZigBee technology. Energy Conversion and Management, 88, 49-59, 2014.

[8] N. C. Batista, R. Melicio and V. M. F. Mendes. Services enabler architecture for smart grid and smart living services providers under industry 4.0. Energy and Buildings, 141, 16-27, 2017.

[9] M. Beaudin, H. Zareipour, A. Schellenberglabe and W. Rosehart. Energy storage for mitigating the variability of renewable electricity sources: an updated review. Energy for Sustainable Development, 14, 302-314, 2010.

[10] P. Denholm, E. Ela, B. Kirby and M. Milligan. The Role of Energy Storage with Renewable Electricity Generation. National Renewable Energy Laboratory, Colorado, USA, 2010.

[11] K. C. Divya and J. Østergaard. Battery energy storage technology for power systems-an overview. Electric Power Systems Research, 79, 511-520, 2009.

[12] M. Glesner and F. Philip. Embedded systems design for smart system integration. In IEEE Computer Society Annual Symposium on VLSI, Natal, Brazil, pp. 32-33. 2013.

[13] I. L. R. Gomes, H. M. I. Pousinho, R. Melício and V. M. F. Mendes. Bidding and optimization strategies for wind-PV systems in electricity markets assisted by CPS. Energy Procedia, 106, 111-121, 2016.

[14] I. L. R. Gomes, H. M. I. Pousinho, R. Melício and V. M. F. Mendes. Stochastic coordination of joint wind and photovoltaic systems with energy storage in day-ahead market. Energy, 124, 310-320, 2017.

[15] I. L. R. Gomes, H. M. I. Pousinho, R. Melício and V. M. F. Mendes. Aggregation platform for wind-PV-thermal technology in electricity market. In IEEE Proceedings of the International Symposium on Power Electronics, Electrical Drives, Automation and Motion, pp. 799-804. 2018.

[16] J. González-Garcia, R. M. R. Muela, L. M. Santos and A. M. González. Stochastic joint optimization of wind generation and pumped-storage units in an electricity market. IEEE Transaction on Power Systems, 23, 460-468, 2008.

[17] T. V. Graaf. Is OPEC dead? Oil exporters, the Paris agreement and the transition to post-carbon world. Energy Research and Social Science, 23, 182-188, 2017. 
[18] K. W. Hedman and G. B. Sheble. Comparing hedging methods for wind power: using pumped storage hydro units vs. options purchasing. In IEEE International Conference on Probabilistic Methods Applied to Power Systems, Stockholm, Sweden, pp. 1-6. 2006.

[19] R. G. Kavasseri and K. Seetharaman. Day-ahead wind speed forecasting using f-ARIMA models. Renewable Energy, 34, 1388-1393, 2009.

[20] R. Laia, H. M. I. Pousinho, R. Melicio and V. M. F. Mendes. Self-scheduling and bidding strategies of thermal units with stochastic emission constraints. Energy Conversion and Management, 89, 975-984, 2015.

[21] R. Laia, H. M. I. Pousinho, R. Melicio and V. M. F. Mendes. Bidding strategy of wind-thermal energy producers. Renewable Energy, 99, 673-681, 2016.

[22] R. Laia, H. M. I. Pousinho, R. Melício and V. M. F. Mendes. Bidding decision of wind-thermal GenCo in day-ahead market. Energy Procedia, 106, 87-96, 2016.

[23] M. Lang. From industry 4.0 to energy 4.0. future business, models and legal relations. In Digitalisierung in der Energiewirtschaft XX. Jahrestagung Institut für Berg- und Energierecht, 2016.

[24] H. Leng, X. Li, J. Zhu, H. Tang, Z. Zhang and N. Ghadimi. A new wind power prediction method based on ridgelet transforms, hybrid feature selection and closed-loop forecasting. Advanced Engineering Informatics, 36, 20-30, 2018.

[25] Y. Liu, J. Shi, Y. Yang and W. J. Lee. Short-term wind power prediction based on wavelet transform-support vector machine and statistic characteristics analysis. IEEE Transaction on Industry Applications, 48, 1136-1141, 2012.

[26] P. Louka, G. Galanis, N. Siebert, G. Kariniotakis, P. Katsafados, I. Pytharoulis and G. Kallos. Improvements in wind speed forecasts for wind power prediction purposes using Kalman filtering. Journal of Wind Engineering and Industrial Aerodynamics, 96, 2348-2362, 2008.

[27] F. Martínez-Álvarez, A. Troncoso, G. Asensio-Cortés and J. C. Riquelme. A survey on data mining techniques applied to electricity-related time series forecasting. Energies, 8, 13162-13193, 2015.

[28] J. Matevosyan and L. Söder. Minimization of imbalance cost trading wind power on the shortterm power market. IEEE Transactions on Power Systems, 21, 1396-1404, 2006.

[29] G. Rajgor. Greater acceleration of renewables required to meet COP21 goal. Renewable Energy, 17, 175-177, 2016.

[30] S. Sundararagavan and E. Baker. Evaluating energy storage technologies for wind power integration. Solar Energy, 86, 2707-2717, 2012.

[31] A. Torres-Barrán, A. Alonso and J. R. Dorronsoro. Regression tree ensembles for wind energy and solar radiation prediction. Neurocomputing, 326-327, 151-160, 2019.

[32] T. Vandyck, K. Keramidas, B. Kitous and Z. Vrontisi. A global stocktake of the Paris pledges: implications for energy systems and economy. Global Environmental Change, 41, 46-63, 2016.

[33] Red Eléctrica de España - Esios. 2017. https://www.esios.ree.es/es. 